\title{
Management of Congenital Bile Duct Cysts
}

\author{
Jean-Yves Mabrut ${ }^{\mathrm{a}} \quad$ Guillaume Bozio $^{\mathrm{a}} \quad$ Catherine Hubert $^{\mathrm{b}}$ Jean-François Gigot ${ }^{\mathrm{b}}$ \\ ${ }^{a}$ Croix-Rousse University Hospital, Lyon, France; bSaint-Luc University Hospital, Brussels, Belgium
}

\section{Key Words}

Bile duct cyst $\cdot$ Surgery $\cdot$ Liver transplantation $\cdot$ Caroli's

disease $\cdot$ Todani classification

\begin{abstract}
Bile duct cysts $(B D C)$ are rare congenital anomalies of the biliary tree that are characterized by cystic dilatation of the extrahepatic and/or intrahepatic bile ducts. Presence of an anomalous bilio-pancreatic junction allowing pancreatic juice to reflux into the biliary tree is the most widely aetiopathogenic concept currently accepted. BDC are associated with biliary stasis and lithiasis, and the whole biliary epithelium is considered at risk of malignant transformation. Magnetic resonance cholangio-pancreatography is currently the most accurate preoperative imaging study to assess cyst anatomy and classify the disease according to the standard Todani classification. Complete cyst excision with cholecystectomy followed by biliary reconstruction using a Roux-en$Y$ hepatico-jejunostomy is the treatment of choice for the extrahepatic component of the disease (type I and IV BDC), but cystic involvement up to the roof of the main biliary convergence represents a real surgical challenge to perform complete resection. The optimal treatment of intrahepatic bile duct dilatations remains controversial, especially for type IV-A BDC. In type V BDC (Caroli's disease), extent of the liver resection is tailored to intrahepatic disease extent and takes into consideration the presence and severity of under-
\end{abstract}

lying chronic liver (congenital hepatic fibrosis) and kidney disease. Late postoperative cholangitis secondary to intrahepatic strictures and lithiasis, as well as the risk of metachronous cholangiocarcinoma that can occur throughout the whole biliary tree even after complete cyst excision, represent serious complications justifying careful long-term follow-up.

Copyright $\odot 2010$ S. Karger AG, Basel

\section{Demographics}

Bile duct cysts (BDC) is a rare congenital disorder, with an estimated prevalence ranging from 1 in 13,500 to 1 in 200,000 live births in Western countries, while the disease is more frequent in Asia [1-4]. Disease occurrence after childhood is uncommon. Indeed, about $25 \%$ of BDC are diagnosed antenatally or within the first year of life, $60 \%$ during the first decade of life, and about $20 \%$ go undiagnosed into adulthood $[2,3]$. However, in USA and Europe, an increasing number of adult patients are reported. This trend might reflect an institutional referral bias or the lack of attention to the disease in regions with a low prevalence, but might also possibly be due to improved accuracy of non-invasive imaging studies [5-7]. A marked female predominance has been widely recognized (female to male ratio 3:1) [2,3].

\section{KARGER}

Fax +4161306 1234

E-Mail karger@karger.ch

www.karger.com
(C) 2010 S. Karger AG, Basel

$0253-4886 / 10 / 0271-0012 \$ 26.00 / 0$

Accessible online at:

www.karger.com/dsu
Prof. Jean-François Gigot, Division of Hepatobiliary and Pancreatic Surgery

Department of Abdominal Surgery and Transplantation

Saint-Luc University Hospital

Hippocrate Avenue, 10, BE-1200 Brussels (Belgium)

Tel. +32 276414 61, Fax +32 276489 18, E-Mail gigot@chir.ucl.ac.be 

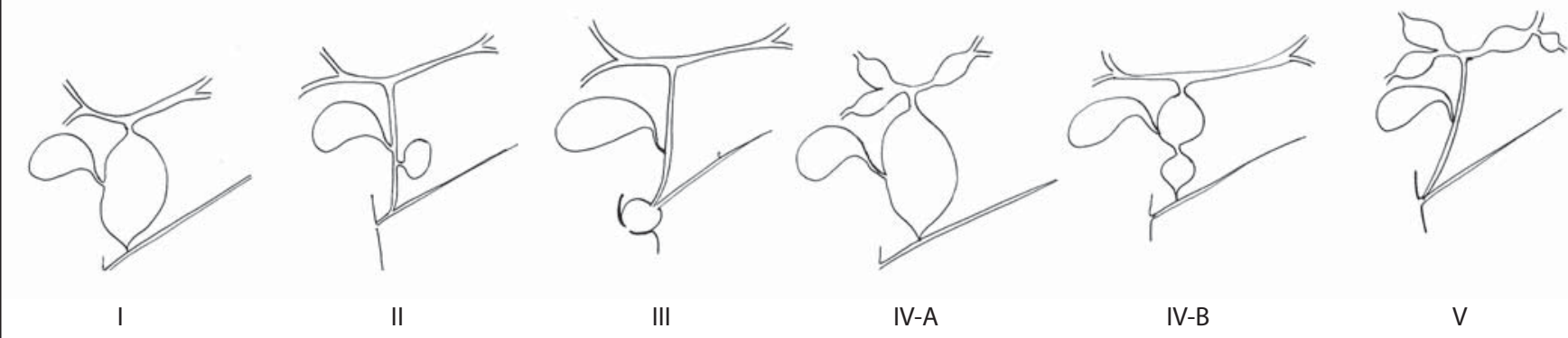

Fig. 1. Various types of BDC according to Todani's classification.

\section{Classification}

BDC are classified according to the site (extrahepatic and/or intrahepatic), extent (segmental or complete) and shape (cystic or saccular, fusiform) of the biliary anomaly. Dilatation may occur anywhere throughout the biliary tree. The Todani classification, used as a standard [8] (fig. 1), defined type I (I-A: cystic, I-B: segmental, I-C: fusiform shape) as dilatation of the extrahepatic biliary tree, type II as diverticulum of the supraduodenal extrahepatic biliary tree, type III as dilatation of the intraduodenal portion of the extrahepatic biliary tree (choledochocele) and identified two subtypes IV: type IV-A with both intra- and extrahepatic dilatation of the biliary tree and type IV-B with multiple and segmental dilatations of the extrahepatic biliary tree. Type V BDC, originally described by Caroli [2], corresponds to dilatation strictly limited to the intrahepatic biliary tree without extrahepatic component. Unilobar extension of type $\mathrm{V}$ BDC is more frequently encountered (80\%) in surgical series, especially on the left side of the liver, but the disease may diffusely involve the entire intrahepatic biliary tree [9]. Type I BDC is the more frequent cyst type encountered (70-90\%), followed by type IV-A (10-20\%), choledochocele (4\%), type II (2-5\%) and type V (1\%). The distribution of BDC types is similar in adults and children, with the exception of Todani type IV-A BDC which are more frequent in adults [2,3]: an incidence of $18.9 \%$ was reported by Yamaguchi [4] in his collective Japanese review but ranges from 35 to $83 \%$ in recently reported adult series $[2,3,10]$. The higher rate of type IV-A BDC in adult patients suggests that, although of a congenital origin, these lesions may progress with time or may develop later in life.

Bile Duct Cysts

\section{Physiopathology}

Currently, there is no universally accepted aetiology of BDC. For types I-IV BDC, several theories have been proposed, but the most widely accepted hypothesis is the long common channel theory of Babbitt [2,3]. An anomalous bilio-pancreatic junction (ABPJ) with a common channel longer than $10-15 \mathrm{~mm}$ and a pancreatico-biliary junction proximal to the sphincter of Oddi may allow pancreatic juice to reflux into the biliary tree, resulting in increased intraductal pressure that may lead to inflammation and secondary ductal dilatation $[2,3]$. This hypothesis is supported by the detection of high concentrations of pancreatic enzymes in the bile of these patients. The incidence of ABPJ ranges from 96 to $100 \%$ in paediatric series and from 68 to $94 \%$ in adult series [2, 3]. ABPJ may be associated with pancreatic stones or protein plug in the dilated common channel or accessory pancreatic duct leading to increased bile duct obstruction and incidence of acute pancreatitis in these patients $[5,11]$. Anatomical or functional obstruction of the distal part of the extrahepatic biliary tree has also been reported as a possible pathophysiological precursor of BDC. Furthermore, the hypothesis of oligoganglionosis in the distal part of the extrahepatic biliary tree has also been advocated. This may result in inadequate bile duct autonomic innervation responsible for dysmotility and functional obstruction of the common bile duct (equivalent to achalasia of the oesophagus or Hirschsprung's disease of the colon) [2].

Intrahepatic BDC, corresponding to type $\mathrm{V}$ in the Todani classification, results from embryological malformation of the ductal plate [12]. These anomalies are responsible for bile ductular proliferation in the ductal plate configuration, which may be apparent microscopically or 
macroscopically. If the large intrahepatic bile ducts are affected, this results in Caroli's disease, whereas diseased small interlobar bile ducts result in congenital hepatic fibrosis. If all levels of the biliary tree are affected, features of both congenital hepatic fibrosis and Caroli's disease are present, this condition having been named 'Caroli's syndrome' and might be associated with kidney disease (from tubular ectasia to polycystic kidney disease) [12].

\section{Clinical Presentation}

BDC may remain asymptomatic for many years and diagnosis may be obtained in asymptomatic patients on imaging studies for an unrelated process. When symptomatic, the most common symptoms mimic biliary lithiasis. The classic triad of jaundice, right upper quadrant pain and a palpable abdominal mass is much more often encountered in children than in adults ( $85 \mathrm{vs.} 25 \%$ respectively), but when an abdominal mass is found in adults, biliary malignancy must be suspected [3]. Abdominal pain, pancreatitis, or a history of cholecystectomy for presumed biliary symptoms is more common in adults and cholecystitis, cholangitis and pancreatitis tend to occur at a somewhat higher rate $(20-60 \%)$ than in children in the literature $[6,13]$. Complicated clinical presentation (cholangitis, liver abscess, biliary cirrhosis) is more frequent in adults than in young patients and in patients with previous cyst drainage procedures, confirming thus the prohibitive late complication rate encountered after cyst-enteric drainage operations $[3,5]$. The mean time elapsed from the development of symptoms to diagnosis and treatment appears to be significantly longer in adults than in children and was estimated for adult patients to be up to 6 years according to the literature $[6,14]$.

\section{Associated Hepatobiliary and Pancreatic Diseases}

Coexistent hepatobiliary and pancreatic (HBP) diseases are frequently observed in adult BDC patients and in patients who have undergone previous cyst-enteric drainage procedures $[2,3]$. Stone formation secondary to bile stasis is the most frequent condition occurring in adults, affecting from 37.5 to $74 \%$ of BDC patients [5]. Hepatolithiasis is most often reported in type IV-A BDC patients [2], sometimes related to frequent membranous or septal stenosis of the segmental bile duct near the main biliary convergence [15]. The risk of pancreatitis that is reported in $30-70 \%$ of adult BDC patients increases markedly with cyst size and in the presence of ABPJ $[4,6]$. Obstruction of the pancreatico-biliary common channel by stones or plug protein impaction, leading to increased pressure in the pancreatic duct, is postulated as the significant precipitating factor in spite of bile reflux in the pancreatic duct. Situations associated with increased intra-abdominal pressure in adulthood, such as pregnancy, may also rarely predispose to spontaneous cyst rupture and biliary peritonitis [3].

Half of the cases of type V BDC are associated with coexistent congenital hepatic fibrosis that corresponds to an inherited congenital malformation transmitted as an autosomal recessive trait [12]. The main consequence is portal hypertension responsible for digestive haemorrhage from oesophageal variceal bleeding.

\section{Previous HBP Treatments}

A previous HBP procedure including cholecystectomy or other surgical biliary exploration before diagnosis is commonly reported in the adult population (10-50\%) [57]. Moreover, patients might also be referred for incomplete resection of the distal part of the extrahepatic dilatation leading to recurrent pancreatitis. The presence of coexisting HBP disease and the performance of previous biliary surgery account for the more complex management of the disease required in adult patients [5].

\section{Radiological Assessment}

Preoperative imaging is essential to establish the diagnosis of BDC, classify the extent of the disease and plan surgical strategy. BDC can now be diagnosed at any age of life, including antenatally by ultrasonography. Precise identification of the type, extent of the biliary tree dilatation and information on the pancreatic and bile duct anatomy and disease are essential to plan surgical strategy. Percutaneous transhepatic and intraoperative cholangiography are considered useful for assessing cyst anatomy and extent of intrahepatic disease in type IV-A cyst, for identifying associated biliary tract anomalies and diseases such as hepatolithiasis, ductal stricture or carcinoma. Endoscopic retrograde cholangio-pancreatography (ERCP) is an accurate invasive technique for the definition of the anomalous pancreatico-biliary ductal junction, this information being important to avoid injury of the pancreatic duct during distal cyst excision and to determine the need for additional treatment of associ- 


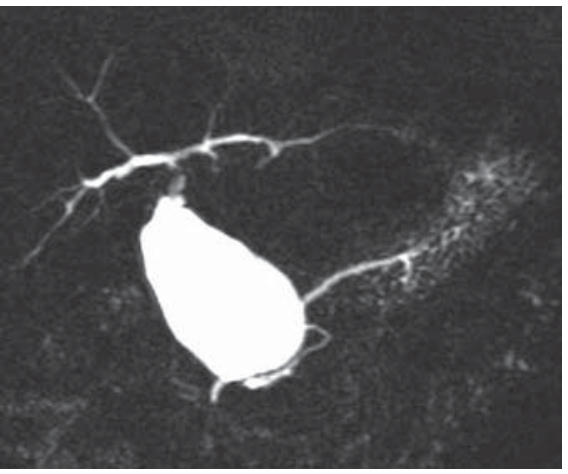

Fig. 2. Magnetic resonance cholangio-pancreatography: Todani type I BDC without main biliary convergence involvement.

ated pancreatic ductal stones or stricture. However, the current 'gold standard' for staging BDC is magnetic resonance cholangio-pancreatography (MRCP) [3] (fig. 2). The main advantage of MRCP is its non-invasive nature and its ability to assess cyst anatomy, to identify size, site, and shape of bile duct dilatation and to detect ABPJ, without the risk of pancreatitis or cholangitis sometimes encountered with ERCP [16]. Reliable diagnosis of Caroli's disease is based on typical radiological features. By definition, these cystic intrahepatic cavities are communicating with the intrahepatic biliary tree. An additional radiological feature is the presence of enhancing fibrovascular bundles (corresponding to septa containing portal veins and hepatic arteries) protruding into the lumen of the ectatic intrahepatic bile ducts. This specific but inconstant feature at liver imaging was defined by Choi et al. [17] as the 'central dot sign'.

\section{Risk of Malignant Transformation}

A significant association between BDC and HBP malignancy is unanimously reported with a clear increasing age-related incidence. Cholangiocarcinoma (CC) is the most frequent histological type encountered. The incidence of synchronous CC associated with BDC is estimated to be $2.5-30 \%[2,3]$ and was $6 \%$ in the largest reported Western experience [13]. Todani et al. [18] collected data from 73 institutions in Japan and reported an incidence of $17.5 \%$ that is higher than the $0.01-0.38 \%$ incidence found in large autopsy series in the normal population. The risk of developing CC in BDC patients is estimated to be 20 - to 30 -fold higher (up to 120) than the general population and CC occurs in younger patients (at a mean age of 32 years), about two decades earlier than in the general population [3]. Long-term survival is rare, even after surgical excision, but the prognosis is the same as for $\mathrm{CC}$ in the general population.

In BDC patients, the age-related incidence of cyst-associated cancer has been shown to increase from $0.7 \%$ in the first decade of life to $14.3 \%$ after the age for 20 years [19]. In the series of Komi et al. [20], the overall incidence of malignancy was $0.3 \%$ in children compared to $15.6 \%$ in adults with again an age-related incidence: $26 \%$ in patients aged $>40$ years and $45.5 \%$ in those aged $>70$ years.

The whole biliary epithelium is considered at risk of malignant transformation and malignancy may arise either in the cystic dilatation (50\%) or in remnant tissues or non-dilated parts of the biliary tree $[8,11]$. Coexistent hepatic or pancreatic malignancies have also been reported. CC may develop in all types of BDC, but type I and especially type IV-A BDC show a greater incidence.

Out of the 241 cases of carcinoma collected by Todani et al. [18], 45 (18.6\%) were encountered following previous cyst-enteric drainage procedures. In that series, the mean interval between the biliary bypass procedure and the detection of carcinoma was 10 years. These features support the recommendation by Stain et al. [14] that low-risk patients, even asymptomatic, should undergo elective excision of BDC even previously treated by cyst-enterostomy.

\section{Surgical Treatment}

Features to consider for optimal surgical treatment of BDC patients include patient's age, symptoms and complications, coexistent HPB diseases, previous cyst-related surgical procedures, cyst type on imaging studies, and age-related risk for synchronous malignancy. To date, management of BDC is dictated by cyst location but not by cyst configuration (cystic or fusiform dilatation). Based on the substantial lifetime risk of developing CC, complete cyst excision is considered to be the standard treatment.

\section{Type I BDC}

Complete cyst excision of the extrahepatic component of BDC combined with cholecystectomy, followed by Roux-en-Y biliary reconstruction, is considered to be the treatment of choice for type I and IV BDC $[2,3]$. A cholecystectomy is recommended due to the high risk for gallbladder malignancy, particularly associated when $\mathrm{ABPJ}$ is present [21]. Treatment of BDC by cyst-enterostomy (cyst-duodenostomy or cyst-jejunostomy) is no lon- 


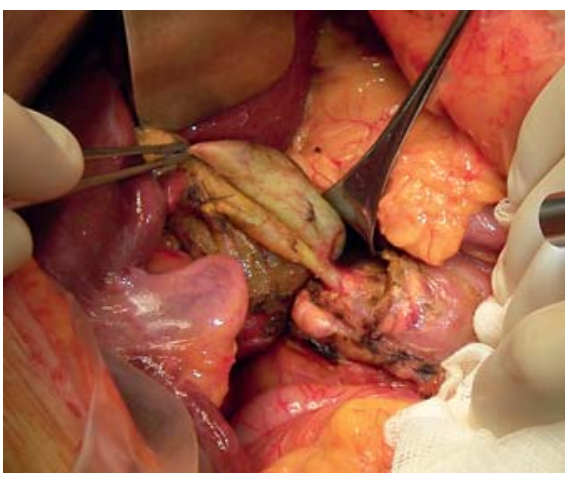

Fig. 3. Peroperative view: intrapancreatic complete distal excision of type I BDC (same patient as in fig. 2).

ger recommended $[2,3]$. Indeed, $\mathrm{BDC}$ are associated with the development of both CC and gallbladder cancer, and enteric drainage without excision does not diminish this malignant potential. Moreover, the drainage provided by a cyst-enteric anastomosis is associated with biliary stasis and recurrent cholangitis. As a result, stricture formation is common (12-73\%) and stasis and cholangitis also lead to malignant transformation [5-7, 14].

In contrast, complete $\mathrm{BDC}$ excision appears to be the treatment of choice and can currently be performed safely either as a primary or a secondary procedure (after failure of internal drainage). Complete cyst excision means that the division of the biliary tract should be carried out in healthy biliary tissue, either at the superior and inferior part of the cystic component, with the use of frozensection pathological examination to rule out the presence of cancer. We recently emphasized that cystic involvement up to the roof of the main biliary convergence or the secondary biliary divisions represented a real surgical challenge for complete cyst excision, leading to perform incomplete resection and reconstruction on non-healthy bile ducts [22]. In such cases, a clear demarcation between non-dilated peripheral intrahepatic (hepatic or sectorial) bile ducts at their level of entrance into a dilated and diseased main or secondary biliary division allows differentiating these BDC type from those defined by Todani et al. [23] as type I-C BDC. The latter indeed present a secondary continuous extent of fusiform dilatation of the choledochus to peripheral intrahepatic bile ducts and such intrahepatic biliary dilatation returns to a normal size after surgical excision of the extrahepatic component of $\mathrm{BDC}$, thus not requiring resection.

At their distal part, type I and IV BDC require excision of the intrapancreatic cystic portion down to the healthy distal bile duct, if any (fig. 3). In this setting, precise preoperative knowledge of ABPJ anatomy is a key factor to avoid intraoperative damage of the pancreatic duct or of the long common channel during cyst excision.

Concomitant pancreatoduodenectomy is indicated only in patients with malignancy but may be required in case of severely inflamed benign cyst imbedded [7] in the pancreatic head or in case of reoperation of symptomatic huge distal cyst remnant. An alternative to pancreatic resection may be the transection of the cyst by leaving an 'egg-cup bottom' that will then be removed by intramural dissection in order to limit postoperative pancreatic complications.

Biliary reconstruction is performed by using a long 60-cm defunctionalized Roux-en-Y jejunal loop with a wide bilio-digestive anastomosis at the level of the common hepatic duct or, more frequently, of the main biliary convergence after having extended the opening of both hepatic ducts (according to the Hepp-Couinaud technique), a trick reducing the risk of late anastomotic stricture and providing satisfactory long-term results $[13,24]$.

\section{Type II BDC}

Complete but limited cyst excision of type II BDC is indicated. Extrahepatic bile duct resection may be necessary but only in case of a large neck of the cyst at its junction with the common bile duct and it is not routinely recommended [2]. However, the presence of an ABPJ exposes the patient to an increased risk of malignancy in both the gallbladder and bile duct, and a prophylactic excision of the gallbladder is thus advised [21].

\section{Type III BDC}

Due to the exceptional risk of malignancy in type III $\mathrm{BDC}$, transduodenal cyst excision is currently replaced by conservative endoscopic sphincterotomy that is recommended in a cyst $<2 \mathrm{~cm}$ in diameter [2]. Pancreaticoduodenectomy remains indicated in case of coexistent cancer [13].

\section{Type IV-A}

As for type I BDC, complete surgical excision of the extrahepatic component of type IV-A and IV-B BDC is the standard of care. First of all, intraoperative endoscopic exploration of intrahepatic bile ducts should be used to detect intraductal stenosis by membranes or septa at the confluence of the major bile ducts [15]. If any, membranous or bridge-like stenosis should be excised circumferentially, but the management of the intrahepatic component of type IV-A BDC remains controversial. Some au- 
thors are in favour of conservative management, while others promote liver resection, especially in case of left unilobar intrahepatic cystic disease, leading thus to complete extra- and intrahepatic disease excision. Furthermore, in type IV-A BDC complicated by ductal strictures, intrahepatic stones or abscess, liver atrophy or malignancy liver resection is indicated. However, Lipsett and Pitt [25] recommend preservation of the hepatic parenchyma, even in the presence of hepatic stones and strictures, when the liver is not cirrhotic. In this case, they recommend placement of large-bored silastic transhepatic stents to facilitate postoperative stone extraction. Liver transplantation may offer a more durable solution and has to be discussed in case of diffuse intrahepatic disease complicated by intrahepatic stones and recurrent attacks of cholangitis refractory to medical treatment or in case of secondary biliary cirrhosis.

\section{Type V BDC}

The management of this rare form of BDC is particularly difficult. The extent of the disease (localized or diffuse) into the liver and the presence of concurrent underlying chronic liver (congenital hepatic fibrosis, secondary biliary cirrhosis, CC) or kidney disease are decision-making factors influencing the surgical management. Drainage procedures represent palliative treatment options that are often ineffective in the long term and are associated with recurrent cholangitis [12]. Liver resection is the treatment of choice for localized unilobar intrahepatic disease without associated chronic liver disease, expecting excellent long-term results $[9,26]$. In case of disease involving both lobes of the liver, or associated with portal hypertension from congenital hepatic fibrosis or secondary biliary cirrhosis, results are poor after conservative treatment. Most patients will suffer from chronic recurrent cholangitis, portal hypertension with variceal bleeding, and death secondary to liver failure or carcinoma. These patients should therefore be considered as candidates for a liver transplant. Indeed, transplantation represents the ultimate successful treatment option for complicated forms of the disease (septic complications from diffuse extent of the disease into the liver and fibrosis or cirrhosis responsible for portal hypertension) $[9,27]$ and should be combined with kidney transplantation in case of chronic renal insufficiency. We previously emphasized that treatment at an early stage of the disease, and avoidance of numerous ineffective operative procedures and of an emergency operation in septic patients are key factors for reducing the operative risk and improving long-term results in patients suffering from type V BDC [9].

\section{Late Outcome}

\section{Biliary Complications}

Despite adequate initial treatment, the long-term follow-up of adult patients with BDC is not uneventful. Late postoperative complications include cholangitis, intrahepatic strictures and/or lithiasis, occurring in most cases from anastomotic stricture or biliary stasis in the intrahepatic biliary tree but also in patients with patent anastomotic stoma [6, 14]. Kim et al. [28] recently emphasized that anastomotic stricture tended to increase with increasing cyst size, short symptom duration and the grade of infiltration of inflammatory cells, and was closely related to the type of BDC. They reported a late occurrence of anastomotic stricture and intrahepatic duct stones in $23.5 \%$, which was significantly more frequent in IV-A cysts justifying the need for careful longterm follow-up.

Anastomotic stricture complicating cyst excision can be treated by surgical revision or by a percutaneous transhepatic approach $[6,14,24]$. Hepatolithiasis in segmental intrahepatic dilatations can be treated by transhepatic endoscopic lithotripsy and percutaneous removal but repeated procedures are usually required [6]. Placement of a large-bore silastic transhepatic stent at the time of primary surgery may facilitate postoperative stone extraction [6]. Additionally, confection of a permanent access stoma using a Roux-Y jejunal loop brought to the skin allows easier retrograde access to the intrahepatic biliary tree for further treatment. However, in case of limited and symptomatic intrahepatic bile duct dilatation, liver resection appears to be a more radical treatment $[6,14]$, while liver transplantation is the only curative option in case of diffuse intrahepatic disease complicated by intrahepatic stones and recurrent attacks of cholangitis refractory to medical treatment or in case of secondary biliary cirrhosis [6].

\section{Metachronous Carcinoma}

Metachronous cancer after BDC excision has been reported to develop in the residual portion of BDC after incomplete proximal or distal BDC excision $[29,30]$. However, primary complete cyst excision reduces but does not eliminate the risk of developing malignancy $[2,18,29,30]$. Indeed, metachronous malignancy can develop throughout the whole biliary tree: in non-dilated intrahepatic bile ducts, at the anastomotic level or in the distal intrapancreatic common bile duct. Watanabe et al. [29] emphasized that carcinoma can occur very late, up to 19 years after the initial procedure, justifying lifelong assessment of late complications or subsequent cancer after BDC excision. 


\section{Conclusions}

$\mathrm{BDC}$ are a rare congenital abnormality of the biliary tree that is characterized by cystic dilatation of the extrahepatic and/or intrahepatic bile ducts. Complete cyst excision is currently the treatment of choice for the extrahepatic component of the disease, although the optimal treatment of intrahepatic bile duct dilatations remains controversial, especially for type IV-A BDC. In case of the diffuse intrahepatic form of the disease with intrahepatic stones complicated by cholangitis or in case of associ- ated fibrosis or cirrhosis, liver transplantation has to be discussed. For type V BDC (Caroli's disease), the extent of the liver resection is tailored to that of intrahepatic disease and takes into consideration the presence and severity of underlying chronic liver (congenital hepatic fibrosis) and kidney disease. Due to the age-related risk of synchronous and metachronous CC that can occur throughout the whole biliary tree, even after complete cyst excision, early indication of surgical cyst excision and long-term follow-up are advised.

\section{References}

$>_{1}$ Wiseman K, Buczkowski AK, Chung SW, 11 Sugiyama M, Atomi Y, Kuroda A: Pancreatic Francoeur J, Schaeffer D, Scudamore CH: Epidemiology, presentation, diagnosis, and outcomes of choledochal cysts in adults and in urban environment. Am J Surg 2005; 189 : 527-531.

2 Nagorney DM: Bile duct cysts in adults; in Blumbert LH, Fong Y (eds): Surgery of the Liver and Biliary Tract, ed 3. London, Saunders, 2000, vol 2, pp 1229-1244.

$>3$ Söreide K, Körner H, Havnen J, Söreide JA: Bile duct cysts in adults. Br J Surg 2004;91: 1538-1548.

4 Yamaguchi M: Congenital choledochal cyst. Analysis of 1,433 patients in the Japanese literature. Am J Surg 1980;140:653-657.

5 Gigot JF, Nagorney DM, Farnell MB, Moir C Ilstrup D: Bile duct cysts: a changing spectrum of presentation. J Hepatobiliary Pancreat Surg 1996;3:405-411.

$\checkmark 6$ Lipsett PA, Pitt HA, Colombani PM, Boitnott JK, Cameron JL: Choledochal cyst disease. A changing pattern of presentation. Ann Surg 1994;220:644-652.

7 Jordan PH Jr, Goss JA Jr, Rosenberg WR, Woods KL: Some considerations for management of choledochal cysts. Am J Surg 2004;187:434-439.

$>8$ Todani T, Watanabe Y, Narasue M, Tabuchi K, Okajima K: Congenital bile duct cysts: classification, operative procedures, and review of 37 cases including cancer arising from choledochal cyst. Am J Surg 1977;134: 263-269.

$>9$ Mabrut JY, Partensky C, Jaeck D, Oussoultzoglou E, Baulieux J, Boillot O, et al: Congenital intrahepatic bile duct dilatation is a potentially curable disease: long-term results of a multi-institutional study. Ann Surg 2007;246:236-245.

$>10$ Lenriot JP, Gigot JF, Segol P, Fagniez PL, Fingerhut A, Adloff $\mathrm{M}$ : Bile duct cysts in adults: a multi-institutional retrospective study. French Associations for Surgical Research. Ann Surg 1998;228:159-166. disorders associated with anomalous pancreaticobiliary junction. Surgery 1999;126: 492-497.

12 Nagorney DM: Biliary and liver cysts; in Blumgart LH, Fong Y (eds): Surgery of the Liver and Biliary Tract, ed 3. London, Saunders, 2000, vol 2, pp 1245-1258.

13 Edil BH, Cameron JL, Reddy S, Lum Y, Lipsett PA, Nathan $\mathrm{H}$, et al: Choledochal cyst disease in children and adults: a 30-year single-institution experience. J Am Coll Surg 2008;2006:1000-1008.

14 Stain SC, Guthrie CR, Yellin AE, Donovan AJ: Choledochal cysts in the adult. Ann Surg 1995;222:128-133.

15 Ando H, Ito T, Kaneko K, Seo T: Congenital stenosis of the intrahepatic bile duct associated with choledochal cysts. J Am Coll Surg 1995; 181:426-430.

16 Kim SH, Lim JH, Yoon HK, Han BK, Lee SK, Kim YI: Choledochal cyst: comparison of MR and conventional cholangiography. Clin Radiol 2000;55:378-383.

17 Choi BI, Yeon KM, Kim SH, Han MC: Caroli's disease: central dot sign in CT. Radiology 1990;174:161-163.

18 Todani T, Watanabe Y, Toki A, Urushihara $\mathrm{N}$ : Carcinoma related to choledochal cysts with internal drainage operations. Surg Gynecol Obstet 1987;164:61-64.

19 Voyles CR, Smadja CI, Shands WC, Blumgart LH: Carcinoma in choledochal cysts. Arch Surg 1983;118:986-988.

20 Komi N, Tamura T, Miyoshi Y, Kunitomo K, Udaka H, Takehara H: Nationwide survey of cases of choledochal cyst. Analysis of coexistent anomalies, complications and surgical treatment in 645 cases. Surg Gastroenterol 1984;3:69-73.
21 Kobayashi S, Asano T, Yamasaki M, Kenmochi T, Saigo K, Ochiai T: Prophylactic excision of the gallbladder and bile duct for patients with pancreaticobiliary maljunction. Arch Surg 2001;136:759-763.

22 Mabrut JY, Partensky C, Gouillat C, Baulieux J, Ducerf C, Kestens PL, et al: Cystic involvement of the roof of the main biliary convergence in adult patients with congenital bile duct cysts: a difficult surgical challenge. Surgery 2007;141:187-195.

23 Todani T, Watanabe Y, Toki A, Morotomi Y: Classification of congenital biliary cystic disease: special reference to type Ic and IVA cysts with primary ductal stricture. J Hepatobiliary Pancreat Surg 2003;10:340-344.

24 Myburgh JA: The Hepp-Couinaud approach to strictures of the bile ducts. Injuries, choledochal cysts and pancreatitis. Ann Surg 1993;218:615-620.

25 Lipsett PA, Pitt HA: Surgical treatment of choledochal cysts. J Hepatobiliary Pancreat Surg 2003;10:352-359.

-26 Bockhorn M, Malago M, Lang H, Nadalin S, Paul A, Saner F, et al: The role of surgery in Caroli's disease. J Am Coll Surg 2006;202: 928-932.

27 De Kerckhove L, De Meyer M, Verbaandert C, Mourad M, Sokal E, Goffette P, et al: The place of liver transplantation in Caroli's disease and syndrome. Transplant Int 2006;19: 381-388.

28 Kim JH, Choi TY, Han JH, Yoo BM, Kim JH, Hong J, et al: Risk factors of postoperative anastomotic stricture after excision of choledochal cysts with hepaticojejunostomy. J Gastrointest Surg 2008;12:822-828.

29 Watanabe Y, Toki A, Todani T: Bile duct cancer developed after cyst excision for choledochal cyst. J Hepatobiliary Pancreat Surg 1999;6:207-212.

30 Kobayashi S, Asano T, Yamasaki M, Kenmochi T, Nakagohri T, Ochiai T: Risk of bile duct carcinogenesis after excision of extrahepatic bile ducts in pancreaticobiliary maljunction. Surgery 1999;126:939-944. 